\title{
Predicting Economic Development using Geolocated Wikipedia Articles
}

\author{
Evan Sheehan* \\ Chenlin Meng* \\ Department of Computer Science \\ Stanford University \\ \{esheehan,chenlin\}@stanford.edu \\ Neal Jean \\ Department of Computer Science \\ Stanford University \\ nealjean@stanford.edu
}

\author{
Matthew Tan \\ Department of Computer Science \\ Stanford University \\ mratan@stanford.edu
}

\author{
Marshall Burke \\ Department of Earth System Science \\ Stanford University \\ mburke@stanford.edu \\ Stefano Ermon \\ Department of Computer Science \\ Stanford University \\ ermon@cs.stanford.edu
}

\author{
Burak Uzkent \\ Department of Computer Science \\ Stanford University \\ buzkent@stanford.edu
}

\author{
David Lobell \\ Department of Earth System Science \\ Stanford University \\ dlobell@stanford.edu
}

\begin{abstract}
Progress on the UN Sustainable Development Goals (SDGs) is hampered by a persistent lack of data regarding key social, environmental, and economic indicators, particularly in developing countries. For example, data on poverty - the first of seventeen SDGs - is both spatially sparse and infrequently collected in Sub-Saharan Africa due to the high cost of surveys. Here we propose a novel method for estimating socioeconomic indicators using open-source, geolocated textual information from Wikipedia articles. We demonstrate that modern NLP techniques can be used to predict communitylevel asset wealth and education outcomes using nearby geolocated Wikipedia articles. When paired with nightlights satellite imagery, our method outperforms all previously published benchmarks for this prediction task, indicating the potential of Wikipedia to inform both research in the social sciences and future policy decisions.
\end{abstract}

\section{CCS CONCEPTS}

- Applied computing $\rightarrow$ Economics; • Computing methodologies $\rightarrow$ Neural networks.

\section{KEYWORDS}

deep learning; remote sensing; computational sustainability

\footnotetext{
*Joint first authors.
}

Permission to make digital or hard copies of all or part of this work for personal or classroom use is granted without fee provided that copies are not made or distributed for profit or commercial advantage and that copies bear this notice and the full citation on the first page. Copyrights for components of this work owned by others than ACM must be honored. Abstracting with credit is permitted. To copy otherwise, or republish, to post on servers or to redistribute to lists, requires prior specific permission and/or a fee. Request permissions from permissions@acm.org.

KDD '19, August 4-8, 2019, Anchorage, AK, USA

(C) 2019 Association for Computing Machinery.

ACM ISBN 978-1-4503-6201-6/19/08 . \$ \$15.00

https://doi.org/10.1145/3292500.3330784
ACM Reference Format:

Evan Sheehan, Chenlin Meng, Matthew Tan, Burak Uzkent, Neal Jean, Marshall Burke, David Lobell, and Stefano Ermon. 2019. Predicting Economic Development using Geolocated Wikipedia Articles. In The 25th ACM SIGKDD Conference on Knowledge Discovery and Data Mining (KDD '19), August 4-8, 2019, Anchorage, AK, USA. ACM, New York, NY, USA, 9 pages. https://doi.org/10.1145/3292500.3330784

\section{INTRODUCTION}

The number one goal of the United Nations Sustainable Development Goals is to "end poverty, in all its forms, everywhere." While much has been done to achieve this objective, there still remain vast regions of the world where extreme poverty continues to be a persistent and endemic problem. Progress is hampered by a stubborn lack of data regarding key social, environmental, and economic indicators that would inform research and policy. Many nations lack the governmental, social, physical, and financial capabilities to conduct large-scale data gathering operations and costly on-the-ground surveys to identify and distribute aid to the most needy communities [25]. Unfortunately, this data scarcity is typical of other SDGs as well: healthcare and mortality data [12], infrastructure and transportation statistics, economic well-being and educational achievement information, food security [2] and wealth inequality assessment, among many others. This lack of detailed and consistent information contributes to delayed or suboptimal financial and physical responses from both regional governments and international aid organizations.

There have been numerous attempts to remedy this shortage of socioeconomic information through the combination of machine learning with cheap, globally available data streams such as social media or remotely sensed data. In particular, with regard to healthcare data, [27] use Twitter as an information-rich source from which to track and predict disease levels and the public's concerns regarding pathogens, while [4] pursue a similar task via the analysis of Google trends. [23] mine the tweets of Indonesian citizens in order to understand food shortages as well as analyze food security 

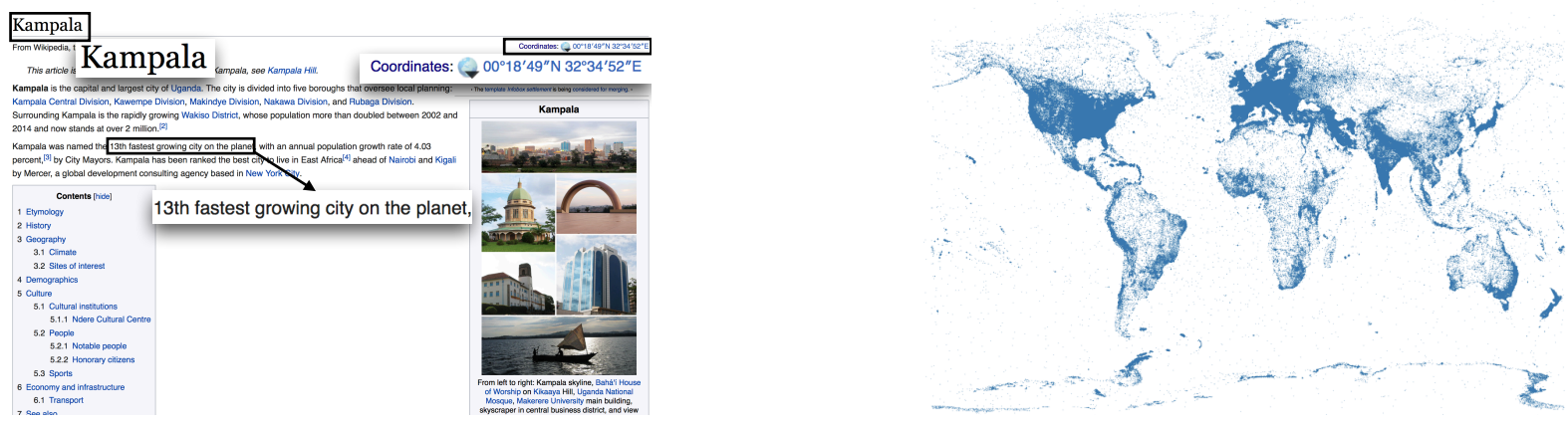

Figure 1: Left: Example of a geolocated Wikipedia article. Articles such as this contain a wealth of information relevant to economic development. Right: Global distribution of geolocated Wikipedia Articles. Note that there is no overlayed basemap, yet the shape of the continents arises naturally from the spatial distribution of articles.

and predict food prices on a granular level. [17] also utilize social media posts to track adverse reactions to drugs with the hope of increasing the scale of data available in the healthcare space. Finally, [3] use mobile phone metadata to attempt to predict poverty levels for data-sparse regions of Rwanda, and [33] successfully perform detailed traffic prediction using the open-source geospatial dataset OpenStreetMap (OSM) [10].

In this paper, we propose the use of Wikipedia as a previously untapped source of data to estimate socioeconomic indicators at very high spatial resolution. Our approach is motivated by both the sheer scale and information-richness of Wikipedia (see Fig. 1 for a spatial distribution of articles), as well as the ease with which its geospatial nature can be exploited. Indeed, in the English Wikipedia alone, there are over 5.7 million entries, each of which averages 640 words in length. Of these, 1.02 million are geolocated (i.e., associated with a physical coordinate) (Fig. 1), allowing them to be used for spatial socioeconomic indicator prediction [26, 29]. Surprisingly, many developing regions of the world contain high concentrations of geolocated articles, with 50,000 for the continent of Africa alone. By providing detailed textual information about locations and entities in an area (e.g., articles about dams, schools, hospitals), we view the articles as data-rich proxies representing the regions around them. In principle, articles like these contain socioeconomic information, though it is often more qualitative than quantitative. Because much of this qualitative data is in unstructured article bodies, and not in infobox/structured formats, it is difficult to extract - this difficulty has prevented Wikipedia from being integrated fully into social science research.

As such, we propose leveraging recent advances in NLP to extract information from free-text Wikipedia articles. We first map geolocated articles to a vector representation using the Doc2Vec method [14]. We then use the spatial distribution of these embeddings to predict socioeconomic indicators of poverty, as measured by ground-truth survey data collected by the World Bank. Using the latent embeddings of nearby geolocated Wikipedia articles to predict the wealth level of geographic locales, we demonstrate that, on its own, Wikipedia is a strong socioeconomic predictor of poverty across 5 Sub-Saharan African nations: Malawi, Nigeria, Tanzania,
Uganda, and Ghana. We further extend our models to incorporate local information about nightime-light intensity as measured by satellites. We then show that this multi-modal approach combining textual information and remotely sensed data significantly improved state-of-the-art results for the task of poverty/economic well-being prediction across these same 5 countries. We achieve Pearson's $r^{2}$ 's of .64, .70, .71, .76, and .76, respectively, as compared to current nightlight state-of-the-art results of $.55, .69, .58$, and .66 (Ghana not included in previous study). Additionally, our approach is able to provide reliable predictions of education-related outcomes, indicating that it can generalize to different types of socioeconomic indicators. We believe the varying data sources and data types in this multi-modal approach provide our models with much more descriptive features. To the best of our knowledge, there has not heretofore been any work done utilizing multi-modal data for poverty prediction. The code for processing nightlights and geolocated Wikipedia articles using the Doc2Vec model can be found in our repository ${ }^{1}$.

\section{DATASETS}

In this section, we describe the ground truth data for the indexes used to measure poverty and education as well as the collection of Wikipedia articles and nightlight images.

\subsection{Socioeconomic data}

Our ground truth dataset consists of data on asset ownership from the Demographic and Health Surveys (DHS) [7] and Afrobarometer [1]. DHS is a set of nationally-representative household surveys conducted periodically in most developing countries, spanning 31 African countries. Afrobarometer (Round 6) is a survey across 36 countries in Africa, assessing various aspects of welfare, education, and infrastructure quality. While data from the DHS and Afrobarometer has been used as training data in multiple machine learning applications $[3,11,19,32]$, it is both costly to create and sparse in its coverage. Typically, the door-to-door surveys used in DHS are conducted approximately every five years, resulting in poor temporal resolution. Each survey also yields only a few thousand

\footnotetext{
${ }^{1}$ https://github.com/ermongroup/WikipediaPovertyMapping
} 

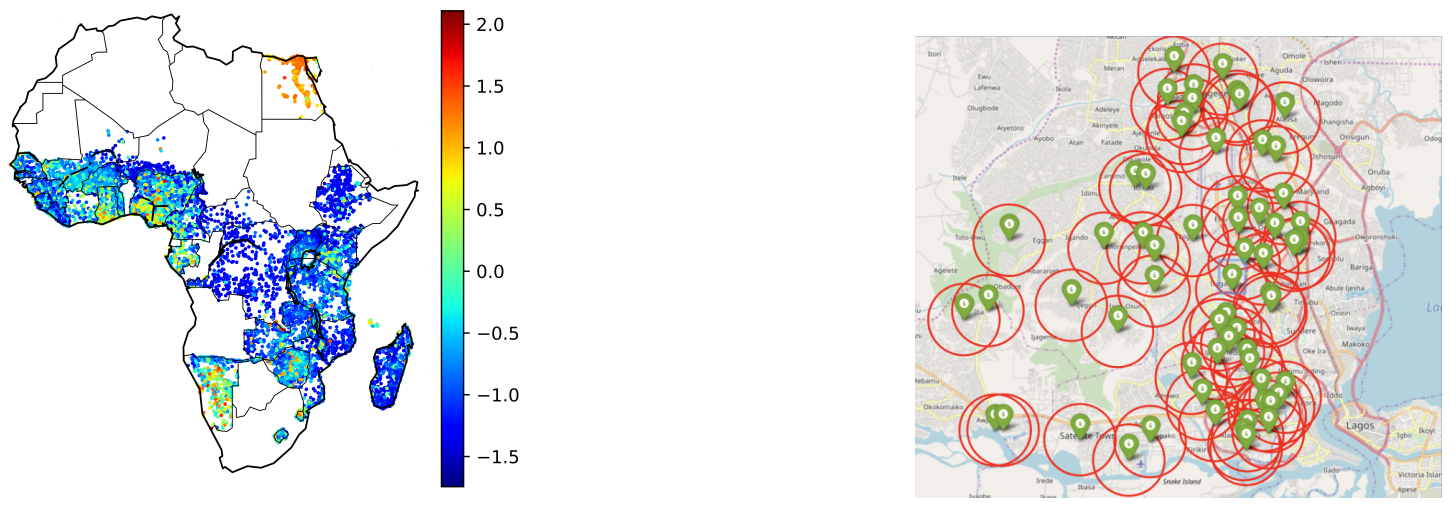

Figure 2: Left: Visualization of ground-truth Asset Wealth Index (AWI) data. Higher values (red) indicate wealthier communities. Right: Jitter in Lagos, Nigeria. Coordinates have up to a $2 \mathrm{~km}$ jitter radius in urban areas and $5 \mathrm{~km}$ in rural ones.

data points per country, even for nations with tens of millions of residents. This means the data captures only a tiny fraction of the population, often leading to entire regions within countries being left out of the survey. Additionally, these surveys are not conducted in every country, and destabilized and dangerous regions of the globe can be excluded due to the hazard presented to interviewers.

From the available DHS survey data, we compute an Asset Wealth Index (AWI) as the first principal component of survey responses to questions about asset ownership (e.g. ownership of bicycles, cars, etc.); this approach is a common one for building a measure of wealth from survey data $[9,28]$. To make this index comparable across all our African countries, we compute the PCA on the data pooled across all countries and survey years, yielding a normalized measure scaled between approximately -2 and 2 (see Fig. 2). We aggregate household-level AWI estimates to the village level - the level at which our latitude and longitude data are available - yielding a training set where labels correspond to a wealth index $y_{i}$ for each lat/lon coordinate $c_{i}$ where the survey was taken. To protect privacy, the recorded coordinate $c_{i}$ for each datapoint provided by DHS contains up to $5 \mathrm{~km}$ of added noise in rural areas and $2 \mathrm{~km}$ of noise in urban areas. This limits the usable spatial resolution of the data. A visualization of this effect is in Fig. 2, right.

To test the effectiveness of our approach on different outcomes, we also use an education index directly from Afrobarometer, capturing the local level of education in a community. The use of Afrobarometer, a survey conducted by a different organization and with a different methodology, allows us to further test the generality of our approach.

\subsection{Geolocated Wikipedia Corpus}

Our input dataset consists of a corpus of geolocated Wikipedia articles $D$. To obtain it, we parsed the June 2018 Wikipedia article dump into its constituent documents and then extracted all those that were geolocated (i.e., associated with a specific latitude and longitude). This process netted over 1 million geolocated articles globally (see Fig. 1 for this distribution as well as an example article) Of note is that throughout Africa there are roughly 50,000 such articles. These geolocated articles are about topics encompassing many categories, including infrastructure (i.e., bridges and roads), populated places, companies, and others. While some articles contain structured information in their infoboxes, many simply contain unstructured text data in their article bodies.

\subsection{Nightlights Imagery}

In addition to learning textual features from geolocated Wikipedia articles, we utilize $(5 \mathrm{~km} \times 5 \mathrm{~km})$ nightlights images compiled by [21]. These images are composed of high-resolution night-time imagery from VIIRS [8] and are of shape $(255,255)$. For each point in the AWI data that we train on, we obtain a nightlights image centered on its coordinate $c_{i}$. The size of these images was set to (5 $\mathrm{km} \times 5 \mathrm{~km}$ ) due to the (maximum) $5 \mathrm{~km}$ noise that each location $c_{i}$ has. Nightlights information has been shown to be highly correlated with economic development [32]. However, nightlights are less effective in the poorest quantiles of the population [11]. In fact, there are numerous locations that are completely dark at night, yet exhibit significant variation in terms of wealth index (see Fig. 6). This motivates the use of alternative covariates for prediction, such as Wikipedia articles.

\section{METHODS}

We consider the task of predicting the wealth level $y_{i}$ at various locations $c_{i}$ (represented by a latitude, longitude pair) using geolocated Wikipedia articles from a suitable neighborhood of $c_{i}$. In order to accomplish this, we use the AWI data described in the previous section as ground truth to train and evaluate our model. Processing these Wikipedia articles poses a large challenge due to the unconstrained textual data they contain. Much of the data is not stored in structured quantitative "infobox" format, but instead in qualitative and textual format in the article bodies. As an example, one can consider the articles on Kampala and Masaka, two different cities in Uganda. In these articles, the infobox data contains only population totals, weather data, elevation, and a few other easily obtained metrics. However, in the unstructured body text, much richer qualitative data is available. For instance, the Kampala article describes construction of a new light rail system in the city, as well as efforts to relocate heavy industry to ease traffic. The Masaka 
article discusses the destruction of the city in the 1980's during a civil war and also lists the most common economic activities of its citizens. These types of facts are likely strongly correlated with the regions' economic well-being, but the data is stored as unstructured text. At the same time, many articles (e.g., about historical events) contain information that is likely not relevant for our predictive task - this makes extracting the relevant information an even more difficult task.

An additional challenge is posed by the biased and censored nature of our Wikipedia corpus. The spatial distribution of articles, their length, and their content vary widely across countries and even across regions within the same country. The fact that there are no articles on hospitals in a region obviously does not mean there is no hospital in that region. Similarly, even if an article is present, it might or might not mention aspects that would be useful for our task. Unfortunately, even the probability that relevant facts (e.g., hospital presence) are mentioned in Wikipedia appears to vary significantly across regions. This makes statistical inference much more difficult. This challenge is of course shared by many other sources of social media data, e.g., Twitter messages. However, it does not arise when using a data source such as nightlights, which are collected uniformly and consistently ${ }^{2}$ across the globe.

Following the proposed framework, we seek to address the following questions: (1) Can we leverage the information in geolocated Wikipedia articles to predict economic indicators, despite the unstructured nature of free text and the inherent bias in the corpus? (2) How does the quality of the predictions compare to conventional approaches based on remote sensing data? (3) Is the information from Wikipedia complementary, i.e., can we develop a multi-modal approach utilizing Wikipedia articles alongside nightlights imagery?

\subsection{Learning Representations of Wikipedia Articles}

The problem of learning suitable representations for unstructured text data has received increasing attention recently [15, 20, 30]. Yet many common methods, such as TF-IDF, Bag-of-Words, and Word2Vec [16], struggle to capture long-range semantic dependencies between words. The Doc2Vec approach [14] remedies these shortcomings by learning robust representations of not only words, but also paragraphs and documents. Doc2 Vec captures long-range word dependencies, word orderings, and holistic semantics of words in a document, and has been used in recent studies [24, 31] that leverage both textual and visual data. Additionally, it has been used on Wikipedia articles to assess the quality of articles $[5,6]$, with an in-depth empirical analysis presented in [13]. Similarly to these works, we use Doc2Vec to embed a Wikipedia article, $D_{i}$, into a $p$ dimensional distributed representation suitable for our downstream task.

To train Doc2Vec, we preprocess the body of 1.02 million geolocated Wikipedia articles, $D$, by tokenizing and lowercasing text and removing extraneous punctuation. This corpus of articles is then fed into a Doc2Vec model that utilizes the Paragraph Vector Distributed Bag of Words (PV-DBOW) training method [14] at the suggestion of [13] and others. The model then learns to project the

\footnotetext{
${ }^{2}$ Bias due to geography is corrected for using physics-based models.
}

articles to $p$-dimensional vector representations. For this task, we choose a window size of 8 , a dimensionality $p$ of 300 , and train the model for 10 epochs - these hyperparameters are similar to those suggested by [13] as being optimal for similar tasks. We denote the function that is parametrized by the Doc2Vec model and returns the $p$-dimensional representation for article $D_{i}$ :

$$
\mathrm{z}_{i}=f^{d 2 v}\left(D_{i}\right)
$$

\subsection{Wikipedia Embedding Model}

We first propose a model to estimate AWI poverty scores $y_{i}$ utilizing only textual information in Wikipedia articles in a neighborhood of $\mathbf{c}_{i}$. Suppose that, for a specific country, we have $M$ points where we have DHS ground survey data. Each of these points corresponds to a location $\mathbf{c}_{i}=\left(c_{i}^{\text {lat }}, c_{i}^{\text {long }}\right)$ where $i \in\{1, \ldots, M\}$. For each $\mathbf{c}_{i}$ we iterate through all the geolocated Wikipedia articles and find $N$ Wikipedia articles $D_{i}^{1}, \ldots, D_{i}^{N}$, each with corresponding location $\left(\mathbf{l}_{i}^{1}, \cdots, \mathbf{l}_{i}^{N}\right)$, that are closest to $\mathbf{c}_{i}$ w.r.t. Euclidean distance. We concatenate the $N$ closest article embeddings $\left(f^{d 2 v}\left(D_{i}^{1}\right), \cdots, f^{d 2 v}\left(D_{i}^{N}\right)\right)$, each of which is a vector in $\mathbb{R}^{300}$. Additionally, we compute their Euclidean distances $(\mathrm{km})$ to $\mathbf{c}_{i},\left(\| \mathbf{1}_{i}^{1}-\right.$ $\left.\mathbf{c}_{i}\|, \cdots,\| \mathbf{l}_{i}^{N}-\mathbf{c}_{i} \|\right)$, and use it as an additional feature. This is to capture the potential lack of articles nearby and allow the model to adjust the predictions as necessary. Thus, the final proposed text embedding can be formulated as:

$$
z_{i}^{t}=\left(f^{d 2 v}\left(D_{i}^{1}\right), \cdots, f^{d 2 v}\left(D_{i}^{N}\right),\left\|\mathbf{1}_{i}^{1}-\mathbf{c}_{i}\right\|, \cdots,\left\|\mathbf{l}_{i}^{N}-\mathbf{c}_{i}\right\|\right)
$$

This provides us with a vector in $\mathbb{R}^{300 \times N+N}$ for each $\mathbf{c}_{i}$. The combination of textual and distance embeddings are then passed through a neural network consisting of a 3 layer Multi-Layer Perceptron (MLP) with sigmoid and ReLU activations. Lastly we perform regression to predict DHS values (e.g., wealth) for the ground truth point $\mathbf{c}_{i}$. A schematic representation of the model is shown in Fig. 3a.

We empirically determine $N$ (the number of closest articles we consider) in our results section (see Fig. 7). Fig. 3 displays the distances to the closest and 10th closest articles for each wealth coordinate $\mathbf{c}_{i}$. Based on these figures, we find that majority of the articles are within $100 \mathrm{~km}$ of their corresponding data point.

\subsection{Multi-Modal Architecture}

Following our textual only model, we propose a novel multi-modal joint architecture that utilizes nightlight imagery and textual information from Wikipedia. For each point $\mathbf{c}_{i}$, we utilize our Wikipedia article features from Eq. 2 as in the previous section. At the same time, we obtain the $(5 \mathrm{~km} \times 5 \mathrm{~km})$ VIIRS nightlight image $x_{i}$ centered at $\mathbf{c}_{i}$. We then pass $x_{i}$ through a convolutional architecture, the embedding space of which can be represented as $z_{i}^{c}=f^{C N N}\left(x_{i}\right)$, where $f^{C N N}$ is parameterized by a deep convolutional network as shown in Fig. 4 and returns $z_{i}^{c} \in \mathbb{R}^{512}$. We then pass $z_{i}^{c}$ through a nonlinear function consisting of two dense layers with ReLU activations obtaining a vector in $\mathbb{R}^{256}$. Next, we concatenate representations from the two different modalities and use another four dense layers, each with a ReLU activation, and a final regression layer to produce the final estimate. The proposed architecture, as shown in Fig. 4, is jointly trained on the task of poverty prediction. 


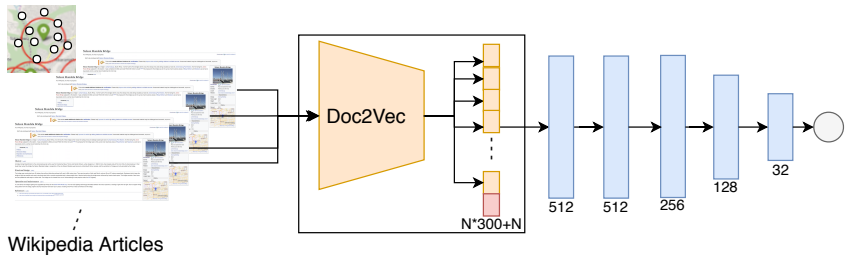

Wikipedia Articles

(a)

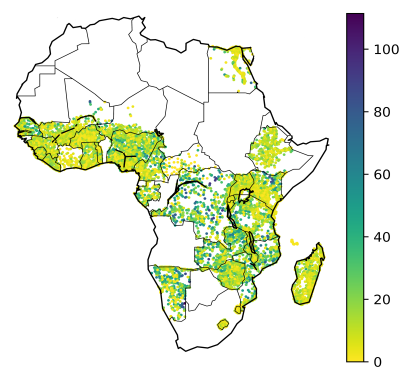

(b)

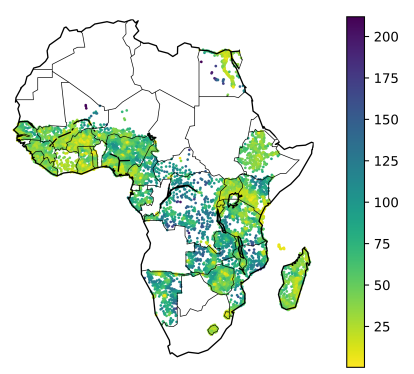

(c)
Figure 3: Top: The proposed Wikipedia Embedding Model is shown in (a). Our architecture uses textual information to regress on economic development. For a given DHS location, we use the $N=10$ closest Wikipedia articles. The blue part is trained on the DHS prediction task, whereas the encoder part of the Doc2Vec is pre-trained on the global geolocated Wikipedia article corpus and fixed during training. Panels (b) and (c) show the average distance $(\mathrm{km})$ to the closest and 10th closest Wikipedia articles for each survey location.

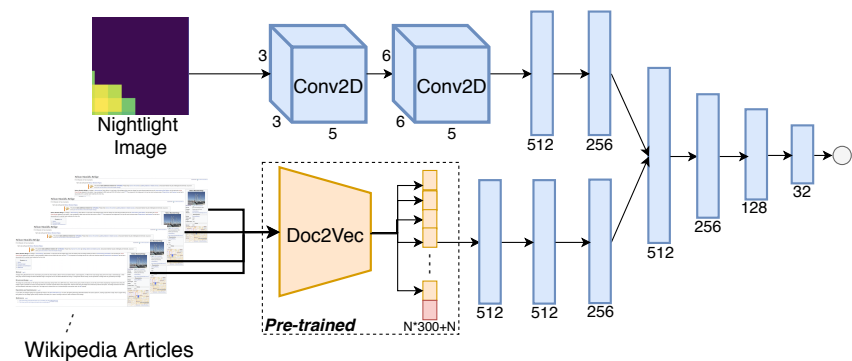

Figure 4: The proposed Multi-Modal architecture. Our architecture uses visual and textual information to regress on survey data. For a given location, we use nightlight imagery and the Wikipedia articles within a certain neighborhood. The blue part is jointly trained on the survey prediction task, whereas the encoder part of Doc2 Vec is pre-trained on the global geolocated Wikipedia article corpus and kept frozen.

However, we freeze the weights of the Doc $2 \mathrm{Vec}$ during training on the poverty task.

\section{POVERTY PREDICTION}

Our study proposes novel single and multi-modal deep neural networks that predict economic development using a combination of
Wikipedia and remotely sensed data (nightlights). To compare with prior work and put our results into perspective, we also consider a baseline Nightlight-Only model, which only uses nightlights as an input. Our Nightlight-Only model is based on the "nightlights branch" of the proposed multi-modal architecture in Fig. 4. This allows us to directly evaluate the contribution of Wikipedia articles in the multi-modal model. We emphasize that this is a strong baseline model, as previous works use simpler, hand-crafted features, such as the mean intensity or simple summary statistics (max, min, median), instead of the image $[18,22]$. Such simpler nightlight models are not included in this study as they significantly underperform our CNN-based Nightlight-Only model. We additionally compare to a model from [11] that uses a combination of nighlights and high-resolution satellite images.

All the architectures are evaluated on three experiments:

(a) Intra-nation tests: Train and test on the same country.

(b) Cross-national boundary testing between African countries: Train on one country and test on another.

(c) Leave-one-country-out training: Train on all countries except one and test on that country, and vice versa.

These experiments were chosen as they reflect the possible realworld applications of this model. In particular, (a) intra-nation inference can be used to fill in gaps of surveys within a country. Data acquired using this method can be very useful in providing local government with the tools to efficiently target aid. Cross-national boundary (b) and leave-one-out based predictions (c), on the other hand, can be used to make predictions in countries with no recent survey data.

As we regress directly on the poverty index, we measure the accuracy of our model using the square of the Pearson correlation coefficient (Pearson's $r^{2}$ ). This metric was chosen so that comparative analysis could be performed with previous literature [11, 21]. Further, in cases of out-of-distribution testing, such as cross-national boundary tests, linear correlation can still be a meaningful metric. For example, our model may have slightly lower predictions if it is trained on a relatively poor country and tested on a wealthy one. In this case, comparing the absolute predicted value with the ground truth would not be a fair option. On the other hand, Pearson's $r^{2}$ is invariant under separate changes in location and scale between the two variables. This allows the metric to still provide insight into the ability of the model to distinguish between poverty levels. In the space of aid, knowing relative wealth indices is often a good alternative to knowing the true ground truth values, as it still provides insight on where to target aid.

\subsection{Wikipedia Embedding Model}

We train our Wikipedia Embedding (WE) model using a Mean Squared Error loss function, along with an Adam optimizer with a learning rate of 0.001 . We report in Table $1 r^{2}$ values obtained from (a) intra-nation (shaded in blue), as well as (b) cross-country and (c) leave-one-out evaluations.

In-Country: We observe that our Wikipedia Embedding (WE) model is able to robustly learn associations with poverty. Compared with the Nightlight-Only (NL) model, we see that WE outperforms NL in all 5 countries. Comparing to the state of the art results from [11], which are based on a combination of nighlights and 


\begin{tabular}{|c|c|c|c|c|c|c|c|c|c|c|c|c|c|c|c|c|c|c|}
\hline \multirow[b]{3}{*}{ Tested on } & \multicolumn{18}{|c|}{ Trained on } \\
\hline & \multicolumn{3}{|c|}{ Ghana } & \multicolumn{3}{|c|}{ Malawi } & \multicolumn{3}{|c|}{ Nigeria } & \multicolumn{3}{|c|}{ Tanzania } & \multicolumn{3}{|c|}{ Uganda } & \multicolumn{3}{|c|}{ All } \\
\hline & $\mathrm{NL}$ & WE & MM & $\mathrm{NL}$ & WE & MM & $\mathrm{NL}$ & WE & MM & $\mathrm{NL}$ & WE & MM & $\mathrm{NL}$ & WE & MM & NL & WE & MM \\
\hline Malawi & 0.30 & 0.40 & 0.48 & 0.24 & 0.49 & 0.64 & 0.34 & 0.35 & 0.55 & 0.37 & 0.42 & 0.56 & 0.34 & 0.25 & 0.52 & 0.40 & 0.38 & 0.56 \\
\hline Nigeria & 0.44 & 0.32 & 0.60 & 0.31 & 0.37 & 0.52 & 0.30 & 0.52 & 0.70 & 0.46 & 0.37 & 0.57 & 0.48 & 0.24 & 0.57 & 0.48 & 0.35 & 0.61 \\
\hline Tanzania & 0.50 & 0.52 & 0.58 & 0.46 & 0.52 & 0.63 & 0.52 & 0.48 & 0.64 & 0.60 & 0.64 & 0.71 & 0.52 & 0.49 & 0.63 & 0.54 & 0.50 & 0.59 \\
\hline Uganda & 0.61 & 0.45 & 0.70 & 0.58 & 0.50 & 0.74 & 0.62 & 0.40 & 0.70 & 0.64 & 0.49 & 0.75 & 0.53 & 0.57 & 0.76 & 0.62 & 0.52 & 0.71 \\
\hline Average & 0.45 & 0.41 & 0.60 & 0.43 & 0.43 & 0.61 & 0.49 & 0.42 & 0.59 & 0.50 & 0.45 & 0.63 & 0.50 & 0.36 & 0.59 & 0.52 & 0.49 & 0.64 \\
\hline
\end{tabular}

Table 1: Pearson's $r^{2}$ values for the Nightlight-Only (NL), Wikipedia Embedding (WE), and Multi-Modal (MM) models on incountry and out-of-country experiments. Columns and rows represent the countries the models were trained and tested on, respectively. The Multi-Modal model outperforms the other models on both in-country (shaded) and cross-country experiments.

high-resolution daytime imagery, we see that we are consistently within $10-15 \%$ of the reported $r^{2}$ 's for training and testing on the same countries, except for Tanzania, where we improve from .58 to .64 .

Out-of-Country: Cross national boundary experiments allow us to test the generalization capabilities of the model. As we are taking $5 \mathrm{~km} \times 5 \mathrm{~km}$ nightlight images of a data point, there may be portions of images similar to both train and test sets because of underlying spatial correlations between the examples. This can also happen for the Wikipedia embeddings, as the $N=10$ closest articles can have some overlap between training and testing examples when evaluating within the same country. Prediction across national boundaries is a more difficult task, both because of covariate shift and because there is almost no spatial correlation between the train and test sets the model can exploit.

The details of these results are in Table 1, where the statistics of a model trained on country A and evaluated on country B are located in the column labelled A and the row labelled B. The "Average" row simply presents the average performance of the models for each column country. For example, Fig. 5 displays the results of the WE model when it is trained on the Ghanaian article distribution and then evaluated on the Tanzanian article distribution. We observe that, while the model may not always predict the correct wealth level for a region, it is often correct in its relative predictions for adjacent regions and regions within the nation in general. This suggests that scaling the model by the mean income level for a country (data which is available) may allow the model to correctly determine both relative and absolute wealth levels.

Comparing WE to NL for out-of-country experiments, we see that NL often outperforms WE by a very slight margin, usually by approximately .1 to .15 in terms of $r^{2}$. This is also true when comparing WE to the out-of-country state of the art results from [11]. The exception to both these trends is that WE outperforms both NL and [11] by about .05 to .1 on over half the models either trained or tested on Malawi. Throughout all these results, the core theme observed is that the WE model is learning generalizable features and not simply picking up on spatial correlations, since the train and test sets are entirely disjoint.

To better understand the role of Wikipedia embeddings, we visualize the predictions of the NL and WE models trained on Ghana and tested on Tanzania in Fig. 6. Because certain countries' nightlights
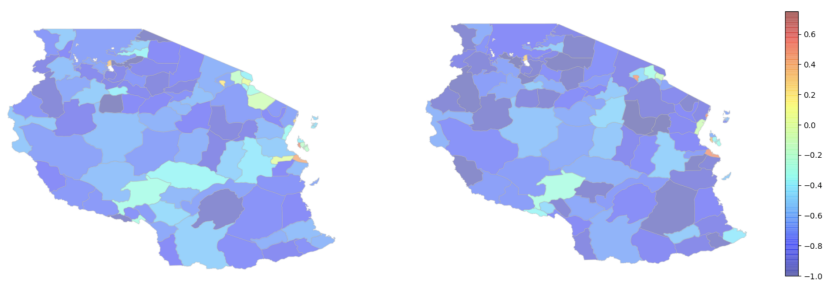

Figure 5: Left: Ground truth values of 1074 data points averaged on Admin 2 Level for Tanzania. Right: Wikipedia Embedding Model predicted values averaged on Admin 2 level for a model trained on Ghana and tested on Tanzania $\left(r^{2}=0.52\right)$. The resulting predictions show that the model is capable of generalizing across national boundaries.

datasets are dominated by dark images, in certain circumstances the Nightlight-Only model is prone to predict constant values, which results in the horizontal line of predictions shown in the left plot of Fig. 6. However, the Wikipedia Embedding model does not suffer from such a shortcoming and is able to increase the range of the predictions, as shown in the right plot of Fig. 6 .

Leave-One-Out: The column "All" in Table 1 represents models trained using the leave-one-out approach, such that a model in column "All" evaluated on nation $B$ was trained on all countries but $B$. The inverse is true for row "All", where a model trained on nation $A$ is tested on all other countries.

Examining the leave-one-out experiments, we observe that training with this approach achieves the best results on average compared to training on any other single country. The average $r^{2}$ of WE models trained in this way is .49, the highest such WE model averages. Interestingly, the models trained on only Tanzania perform similarly or even better than the ones trained on All countries on the Malawi test samples. We hypothesize this is due to similar patterns of the neighboring countries in the dataset.

\subsection{Multi-Modal Model}

We train our Multi-Modal (MM) model using a Mean Squared Error loss function, along with an Adam optimizer with a learning rate of 0.001 . Table 1 displays the Pearson's $r^{2}$ results of the model. 

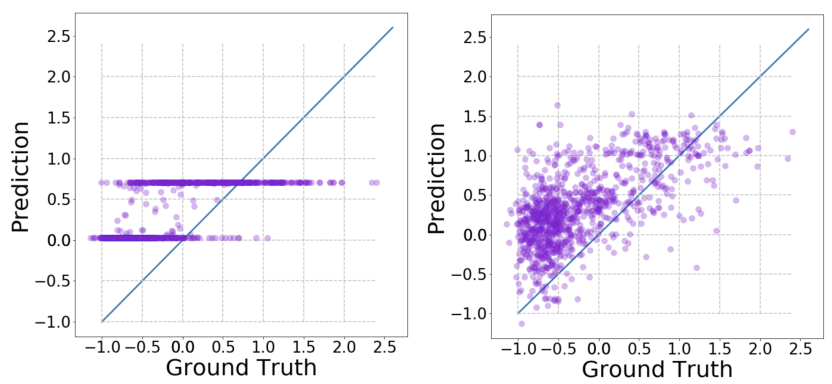

Figure 6: Wealth prediction for Nightlight-Only (Left) and Wikipedia Embedding (Right) models trained on Ghana and tested on Tanzania. Nightlights-Only model produces highly correlated results for different ground points, resulting in horizontal lines. Meanwhile, the Wikipedia Embeddings model leads to a better range of predictions.

Comparing WE with MM, we observe that in general the accuracy $\left(r^{2}\right)$ of the models for out-of-country evaluation improves by almost $50 \%$ across the board for the multi-modal approach. Importantly, the proposed multi-modal architecture outperforms the other models by large margin when trained via the leave-one-out approach, proving that the textual branch maintains its contribution even when training the $\mathrm{CNN}$ with large amounts of nightlights data. Some models, such as the one trained on Uganda and tested on Nigeria, obtain over $100 \%$ improvement, while others, such as the one trained on Ghana and tested on Tanzania, obtain less than a $20 \%$ improvement. Importantly, all train-test country combinations see some degree of improvement. When considering the results of our ablative Nightlight-Only model, we also observe that the MM achieves superior results for both intra-country and cross-boundary tests nearly across the board. Such results suggest that Wikipedia embeddings and nightlight images provide highly complementary information about poverty. Finally, the MM outperforms state of the art by an average of $31.2 \%$ [11], with improvements nearly across the board.

\subsection{Ablation study and Interpretability}

So far we have presented our results for both architectures using $N=10$ closest articles to the ground truth point. To evaluate the sensitivity to $N$, we conducted additional experiments by varying number of articles $N$. As shown in Fig. 7, considering average performance, we can conclude that $N=10$ provides good performance for most of the countries. However, for some countries (Uganda, Tanzania), even a few articles can provide optimal models. We believe this might be due to the different density of articles (see Fig. 3b, and $3 \mathrm{c}$ ) around data points.

To help us better understand Wikipedia article embeddings, we performed a PCA analysis of the learned Wikipedia article embeddings. More specifically, we trained a model on Tanzania, evaluated it on Uganda, ranked all its projections from richest to poorest, and then stored the top third and bottom third of these points, corresponding to the richest $33 \%$ and the poorest $33 \%$ of all projections for Uganda. For each of the points in these wealthiest and poorest

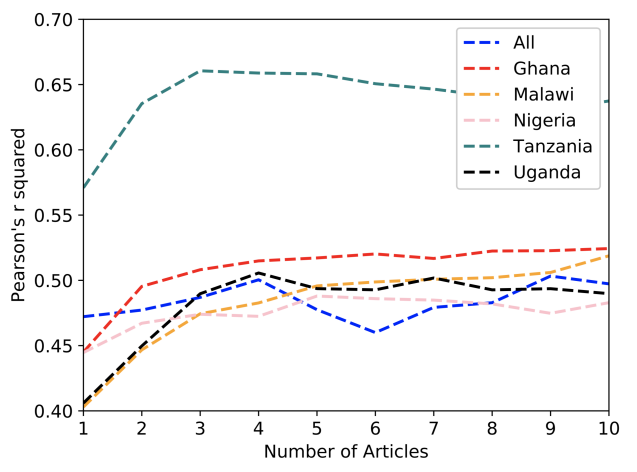

Figure 7: Pearson's $r^{2}$ performance for models trained using varying $N$ geographically nearest article embeddings, trained on the specified country and evaluated on Tanzania.

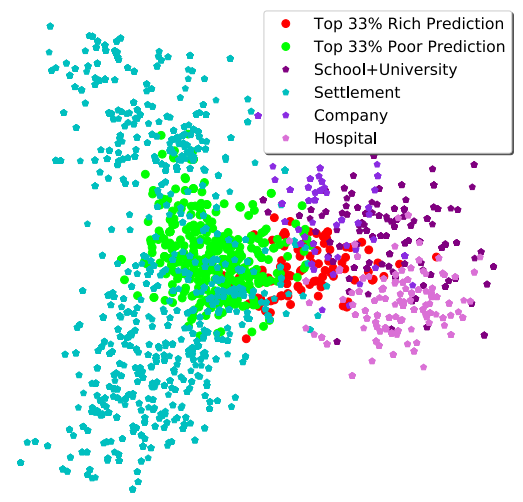

Figure 8: Wikipedia Embedding PCA Analysis. The WE model learns to project the wealthiest $33 \%$ quantile to the space closer to Company, Hospital and School+University related Wikipedia Articles' embeddings.

thirds, we averaged the embeddings of the 10 closest articles to it. We then compared these averaged embeddings' PCA projections to the projections of 'school', 'university', 'hospital', 'company', and 'settlement' articles that appeared in the test set (see Fig. 8). We observed that the average Doc2Vec embeddings of the wealthier coordinates clustered around article categories which intuitively are related to wealth. In particular, wealthier places tended to cluster toward 'school', 'university', 'hospital', and 'company' embeddings, while poorer places were more related to 'settlement' articles.

\section{EDUCATION INDEX PREDICTION}

To further test the effectiveness of our text-only model in predicting other socioeconomic outcomes, we train and evaluate our model on a new task: education level-prediction. Improving the quality of global education is the 4th SDG - access to education is foundational to the creation of self-sustaining and self-improving societies.

In addition to predicting a different type of outcome, we use a new source of ground truth data for this task. We use the Afrobarometer R6 Codebook dataset [1], which is distributed by Afrobarometer, a research network that conducts public attitude surveys 
on democracy, governance, economic conditions, and related issues in more than 35 countries in Africa. We utilize the 'eaeducationlevel' index, which has values ranging from 1 to 4 , with larger number standing for higher level of education (i.e., 1 stands for 'No formal schooling', 4 stands for 'post-secondary qualifications'), for our groundtruth labels. To be consistent with the previous experiments on poverty, we limit our research to 5 countries (see Table 2) in Africa, each with 300 datapoints. Notice that Afrobarometer is sampled at different locations compared to DHS. We evaluate our Wikipedia Embedding model on the (a) Intra-nation and (b) CrossBoundary training tasks, as in the previous section. We use the same training procedure, except we regress on a different outcome.

Table 2 displays our results on this task. We notice that that incountry models (shaded) consistently outperform out-of-country models, as was generally the case for the poverty task. However, on average the performance is worse compared to the poverty task, with averages ranging from .20-.25 compared to .40-.45. One possible explanation is that education is a finer-grained indicator that is more difficult to predict from Wikipedia. We can expect improved results with the inclusion of more education-related articles in future releases. Nevertheless, this experiment suggests that Wikipedia provides useful information to predict multiple socioeconomic indicators, across different countries and even different survey methodologies.

\begin{tabular}{cccccc}
\hline \multicolumn{6}{c}{ Trained on } \\
\hline Tested on & Ghana & Malawi & Nigeria & Tanzania & Uganda \\
\hline Ghana & $\mathbf{0 . 3 1}$ & 0.09 & 0.11 & 0.22 & 0.28 \\
Malawi & 0.21 & $\mathbf{0 . 3 2}$ & 0.09 & 0.23 & 0.24 \\
Nigeria & 0.32 & 0.11 & $\mathbf{0 . 3 9}$ & 0.24 & 0.23 \\
Tanzania & 0.27 & 0.20 & 0.10 & $\mathbf{0 . 3 4}$ & 0.30 \\
Uganda & 0.29 & 0.19 & 0.10 & 0.25 & $\mathbf{0 . 3 0}$ \\
\hline Average & 0.28 & 0.18 & 0.16 & 0.26 & 0.27 \\
\hline
\end{tabular}

Table 2: Performances of Wikipedia Embedding model on education level prediction as measured by Pearson's $r^{2}$. Bolding indicates the best model in each country.

\section{CONCLUSION}

In this paper, we propose a novel method for the utilization of geolocated Wikipedia articles for socioeconomic applications. We do this by obtaining vector representations of articles via the Doc $2 \mathrm{Vec}$ NLP model. We then pair these latent embeddings with survey data and evaluate models to predict poverty and education outcomes: a Wikipedia Embedding model, and a Multi-Modal model that also uses nightlights information. Using this framework, we find Wikipedia articles to be informative about socioeconomic indicators. To the best of our knowledge, this is the first time Wikipedia is used in this way. Additionally, the Multi-Modal model outperforms current state-of-the-art results for the poverty prediction task by over $20 \%$. Our findings suggest this pipeline, and the geolocated Wikipedia article dataset, has applications not just in poverty analysis, but also more general socioeconomic prediction, such as education and health related outcomes. This novel approach could lead to advances in development economics, where studies often rely only on nightlights information. Additionally, we hope this approach will accelerate progress towards the UN SDGs by improving the way we estimate lacking socioeconomic indicators, particularly in developing countries, with the aim of improving responses from regional governments and international aid organizations.

\subsection{Acknowledgements}

This research was supported in part by Stanford's Data for Development Initiative, DARPA World Modelers Program, and NSF grants \#1651565, \#1522054, and \#1733686. 


\section{REFERENCES}

[1] The afro program website. http://http://afrobarometer.org.

[2] Philip Antwi-Agyei, Evan DG Fraser, Andrew J Dougill, Lindsay C Stringer, and Elisabeth Simelton. Mapping the vulnerability of crop production to drought in ghana using rainfall, yield and socioeconomic data. Applied Geography, 32(2):324-334, 2012

[3] Joshua Blumenstock, Gabriel Cadamuro, and Robert On. Predicting poverty and wealth from mobile phone metadata. Science, 350(6264):1073-1076, 2015.

[4] Herman Anthony Carneiro and Eleftherios Mylonakis. Google trends: a webbased tool for real-time surveillance of disease outbreaks. Clinical infectious diseases, 49(10):1557-1564, 2009.

[5] Quang Vinh Dang and Claudia-Lavinia Ignat. Quality assessment of wikipedia articles without feature engineering. In Proc. of the 16th foint Conference on Digital Libraries, pages 27-30. ACM, 2016.

[6] Quang-Vinh Dang and Claudia-Lavinia Ignat. An end-to-end learning solution for assessing the quality of wikipedia articles. In Proc. of the 13th International Symposium on Open Collaboration, page 4. ACM, 2017.

[7] The dhs program website. http://www.dhsprogram.com.

[8] Christopher D Elvidge, Kimberly Baugh, Mikhail Zhizhin, Feng Chi Hsu, and Tilottama Ghosh. Viirs night-time lights. Int. F. Remote Sens., 38(21):5860-5879, November 2017.

[9] Deon Filmer and Lant Pritchett. The effect of household wealth on educational attainment: evidence from 35 countries. Population and development review, 25(1):85$120,1999$.

[10] Mordechai Haklay and Patrick Weber. Openstreetmap: User-generated street maps. Ieee Pervas Comput, 7(4):12-18, 2008.

[11] Neal Jean, Marshall Burke, Michael Xie, W. Matthew Davis, David B. Lobell, and Stefano Ermon. Combining satellite imagery and machine learning to predict poverty. Science, 353(6301):790-794, 2016.

[12] Kathleen Kahn, Stephen M Tollman, Mark A Collinson, Samuel J Clark, Rhian Twine, Benjamin D Clark, Mildred Shabangu, Francesc Xavier Gomez-Olive, Obed Mokoena, and Michel L Garenne. Research into health, population and social transitions in rural south africa: Data and methods of the agincourt health and demographic surveillance system1. Scandinavian fournal of Public Health, 35(69_suppl):8-20, 2007. [13] Jey Han Lau and Timothy Baldwin. An empirical evaluation of doc2vec with prac tical insights into document embedding generation. arXiv preprint arXiv:1607.05368, 2016.

[14] Quoc Le and Tomas Mikolov. Distributed representations of sentences and documents. In International Conference on Machine Learning, pages 1188-1196, 2014 [15] Jimmy Lei Ba, Kevin Swersky, Sanja Fidler, et al. Predicting deep zero-shot convolutional neural networks using textual descriptions. In Proceedings of the IEEE International Conference on Computer Vision, pages 4247-4255, 2015.

[16] Tomas Mikolov, Ilya Sutskever, Kai Chen, Greg S Corrado, and Jeff Dean. Distributed representations of words and phrases and their compositionality. In Advances in neural information processing systems, pages 3111-3119, 2013.

[17] Azadeh Nikfarjam, Abeed Sarker, Karen OâĂŹconnor, Rachel Ginn, and Graciela Gonzalez. Pharmacovigilance from social media: mining adverse drug reaction mentions using sequence labeling with word embedding cluster features. Fournal of the American Medical Informatics Association, 22(3):671-681, 2015.

[18] Abdisalan M Noor, Victor A Alegana, Peter W Gething, Andrew J Tatem, and Robert W Snow. Using remotely sensed night-time light as a proxy for poverty in africa. Population Health Metrics, 6(1):5, 2008.

[19] Barak Oshri, Annie Hu, Peter Adelson, Xiao Chen, Pascaline Dupas, Jeremy Weinstein, Marshall Burke, David Lobell, and Stefano Ermon. Infrastructure quality assessment in africa using satellite imagery and deep learning. In Proc. of SIGKDD, pages 616-625. ACM, 2018.

[20] Liang Pang, Yanyan Lan, Jiafeng Guo, Jun Xu, Shengxian Wan, and Xueqi Cheng. Text matching as image recognition. In AAAI, 2016.

[21] Anthony Perez, Christopher Yeh, George Azzari, Marshall Burke, David Lobell and Stefano Ermon. Poverty prediction with public landsat 7 satellite imagery and machine learning. 112017.

[22] Jeremy Proville, Daniel Zavala-Araiza, and Gernot Wagner. Night-time lights: A global, long term look at links to socio-economic trends. PloS one, 12(3):e0174610, 2017.

[23] UN Global Pulse. Mining indonesian tweets to understand food price crises. fakarta: UN Global Pulse, 2014.

[24] Arnau Ramisa, Fei Yan, Francesc Moreno-Noguer, and Krystian Mikolajczyk Breakingnews: Article annotation by image and text processing. IEEE TPAMI, 40(5):1072-1085, 2018

[25] David E Sahn and David C Stifel. Poverty comparisons over time and across countries in africa. World development, 28(12):2123-2155, 2000.

[26] Evan Sheehan, Burak Uzkent, Chenlin Meng, Zhongyi Tang, Marshall Burke, David Lobell, and Stefano Ermon. Learning to interpret satellite images using wikipedia. arXiv preprint arXiv:1809.10236, 2018.

[27] Alessio Signorini, Alberto Maria Segre, and Philip M Polgreen. The use of twitter to track levels of disease activity and public concern in the us during the influenza a h1n1 pandemic. PloS one, 6(5):e19467, 2011.
[28] Jeroen Smits and Roel Steendijk. The international wealth index (iwi). Social Indicators Research, 122(1):65-85, 2015.

[29] Burak Uzkent, Evan Sheehan, Chenlin Meng, Zhongyi Tang, Marshall Burke, David Lobell, and Stefano Ermon. Learning to interpret satellite images in global scale using wikipedia. arXiv preprint arXiv:1905.02506, 2019.

[30] Liwei Wang, Yin Li, Jing Huang, and Svetlana Lazebnik. Learning two-branch neural networks for image-text matching tasks. IEEE Transactions on Pattern Analysis and Machine Intelligence, 41(2):394-407, 2019.

[31] Qi Wu, Chunhua Shen, Peng Wang, Anthony Dick, and Anton van den Hengel. Image captioning and visual question answering based on attributes and external knowledge. IEEE transactions on pattern analysis and machine intelligence, 40(6):1367$1381,2018$.

[32] Sang Michael Xie, Neal Jean, Marshall Burke, David B. Lobell, and Stefano Ermon. Transfer learning from deep features for remote sensing and poverty mapping. In AAAI, 2016.

[33] Frank F Xu, Bill Y Lin, Qi Lu, Yifei Huang, and Kenny Q Zhu. Cross-region traffic prediction for china on openstreetmap. In Proceedings of the 9th ACM SIGSPATIAL International Workshop on Computational Transportation Science, pages 37-42. ACM, 2016. 